\title{
Early Life Characteristics Associated with Appetite-Related Eating Behaviors in 7-Year-0ld Children
}

\author{
Gabriela Albuquerque, $\mathrm{MPH}^{1}$, Milton Severo, $\mathrm{PhD}^{1,2}$, and Andreia Oliveira, $\mathrm{PhD}^{1,2}$
}

Objective To assess early life characteristics associated with appetite-related eating behaviors in 7-year-old children. Study design The participants are children from the population-based birth cohort Generation XXI. Data on sociodemographics, health, and lifestyles and anthropometrics were collected at birth, and 4- and 7-year-old evaluations. A Portuguese version of the Children's Eating Behavior Questionnaire was completed by mothers $(n=3562$ children) with children aged 7 years old. A 2-factor solution was identified: factor 1-appetite restraint and factor 2 -appetite disinhibition. Associations were estimated through generalized linear models adjusted for maternal age, education, body mass index (BMI) before birth, family structure, number of siblings, and child's sex ( $\beta$ regression coefficients and $95 \% \mathrm{Cls}$ ).

Results Higher appetite restraint at 7 years old was associated with higher maternal age and educational level, families with both parents (1- vs 2 -parent: $\beta=-0.074,95 \% \mathrm{Cl}-0.140,-0.007$ ) and no siblings ( $\geq 2$ vs $0: \beta=-0.152$, $95 \% \mathrm{Cl}-0.224,-0.081)$, and more sedentary lifestyles at 4 years old. It was also associated with lower child and maternal BMI and waist circumference at 4 years old. In contrast, higher appetite disinhibition was associated with lower maternal educational background, having a 1-parent family, more sedentary behaviors $(\geq 120 \mathrm{vs}<120 \mathrm{~min} / \mathrm{d}$ of media: $\beta=0.055,95 \% \mathrm{Cl} 0.018,0.093)$, and higher $\mathrm{BMI}$ and waist circumference at 4 years old.

Conclusions Higher maternal age and education, and a family with both parents at 4 years old seem to influence higher appetite restraint, but less appetite disinhibition at 7 years old. More sedentary lifestyles at 4 years old were associated with higher appetite restraint and appetite disinhibition scores later in childhood. These results can be useful for the development of prevention guidelines and educational strategies aimed at improving healthy eating behaviors. (J Pediatr 2017; 180:38-46).

ating behaviors develop early in life and result from an interplay between genetic predisposition, natural food responses and preferences, and environmental influences. ${ }^{1,2}$ Individual differences in eating behaviors and appetite have been previously associated with differences in children's weight. ${ }^{3-5}$ Obese children show more positive responses toward food, hypothesized to promote food intake, and leaner children express more sensitivity to internal cues of satiety and food fussiness (consumption of an insufficient amount or inadequate variety of food through rejection of food items), ${ }^{6}$ which is likely to reduce food intake. $4,5,7,8$

Some birth-related and early infancy characteristics have been proposed as potential influencers on the development of eating behaviors. Low birth weight, ${ }^{9-11}$ preterm birth, and restrictive intrauterine growth ${ }^{12}$ have been associated with later eating difficulty, pickiness, and a higher risk of low appetite and food enjoyment in childhood. Breastfeeding, hypothesized to promote early self-regulation of energy intake, has been associated with higher satiety responsiveness, ${ }^{13,14}$ in contrast to formula feeding, which is associated with picky eating ${ }^{15}$ (the consumption of an insufficient amount or inadequate variety of food through rejection of food items $s^{6}$ ) and neophobia. ${ }^{16}$

The familial environment also has a strong influence. Children who tend to overeat are more likely to be male, live in a singleparent home, and have both parents overweight or obese. ${ }^{10}$ In this study, family income was associated with both overeating and picky eating. In other studies, younger maternal age and parental financial problems have been identified as risk factors for picky eating, ${ }^{10,15}$ and having siblings has been shown to be both protective ${ }^{6}$ and a risk factor ${ }^{15}$ for developing picky eating. The aim of this prospective study is to assess early life characteristics associated with appetite-related eating behaviors at 7 years old.

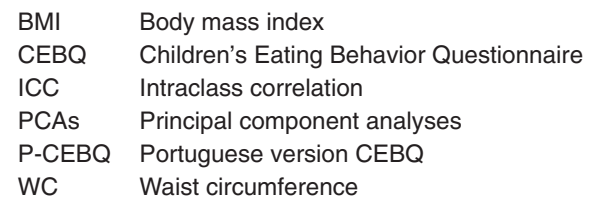

From the ${ }^{1}$ Institute of Public Health-Epidemiology Unit, Universidade do Porto, Porto, Portugal; and ${ }^{2}$ Faculdade de Medicina, Universidade do Porto, Department of Clinical Epidemiology, Predictive Medicine and Public Health, Porto, Portugal

Generation XXI was funded by the Health Operational Program - Saúde XXI, Community Support Framework III, and the Regional Department of Ministry of Health. This study was supported by FEDER (from the Operational Programme Factors of Competitiveness COMPETE), national funding from the Foundation for Science and Technology (Portuguese Ministry of Education and Science; PTDC/SAU-EPI/121532/2010 [FCOMP-01-0124-FEDER-021177]), and the Calouste Gulbenkian Foundation. The authors declare no conflicts of interest.

0022-3476/\$ - see front matter. ๑ 2016 Elsevier Inc. All rights reserved.

http://dx.doi.org10.1016/j.jpeds.2016.09.011 


\section{Methods}

This study includes children from Generation XXI, a prospective population-based birth cohort. ${ }^{17}$ Participants ( 8495 mothers and their 8647 children) were recruited from public maternity units in Porto, Portugal between 2005 and 2006 and were all invited to attend the second (2009-2011) and third (2012-2014) cohort evaluations, when children were aged 4 and 7 years old ( $86 \%$ and $80 \%$ of participation, respectively).

Responses to the Children's Eating Behavior Questionnaire (CEBQ) were available for 5786 children. Of these, 4485 questionnaires were entirely completed, and after retrieving missing data, 5449 were considered valid. Data were recovered in questionnaires that were missing $<50 \%$ of data items, by replacement for the average of the remaining questions within each subdomain of the participant. Spearman-Brown prophecy formula showed that $50 \%$ of the items was enough to obtain a reliability of 0.7 (Appendix; available at www.jpeds.com). The sample was further restricted to 1 twin per family, randomly selected (103 children excluded, $1.19 \%$ of the initial sample), and to participants with complete information on the variables of interest (1887 children excluded, $21.8 \%$ of the initial sample), a total of 3562 participants.

We compared the study sample $(\mathrm{n}=3562)$ characteristics with the remaining cohort $(\mathrm{n}=5085)$ at baseline, and mothers in this study were slightly older ( mean $=29.85 ; \mathrm{SD}=5.22$ vs mean $=28.47 ; \mathrm{SD}=6.26 ; P<.001)$ and more educated $($ mean $=11.27 ; \mathrm{SD}=4.25$ vs mean $=9.88 ; \mathrm{SD}=4.17 ; P<.001)$ than mothers in the remaining cohort.

All the study phases complied with the Ethical Principles for Medical Research Involving Human Subjects expressed in the Declaration of Helsinki. The study was approved by the University of Porto Medical School/S. João Hospital Center ethics committee and a signed informed consent, in accordance with Helsinki, was required for all participants, and signed by their legal guardian.

Data were collected during face-to-face interviews by trained researchers. At baseline, maternal age and education, smoking habits during pregnancy, type of delivery, children's sex, birth weight, and gestational age were recorded. Weight for gestational age was defined according to the sex-specific population-based Kramer growth standards (cut points: 10th and 90th percentiles; standards refer only to single births). ${ }^{18}$

At the 4-year-old evaluation, the number of siblings, family structure, any breastfeeding duration, physical activity, and number of media screening hours (television, computer, or game devices) were asked about. The timing of introduction to complementary feeding and the first food eaten in this period were asked about at 7 years old.

Height, weight, body fat, and waist circumference (WC) were measured at 4 and 7 years old according to standard procedures. The children's body mass index (BMI) was classified according to the age- and sex-specific BMI standard z-scores developed by the World Health Organization ${ }^{19}$ and recoded into under/normal weight (BMI $<2 \mathrm{SD}$ ) and overweight/obese (BMI $\geq 2 \mathrm{SD}$ ). WC was measured at the umbilicus level, to the nearest $0.1 \mathrm{~cm}$. This variable was recoded into tertiles $(<50.5 \mathrm{~cm}$; $50.5-53.5 \mathrm{~cm} ; \geq 53.6 \mathrm{~cm}$ ). Bioelectric impedance analysis was performed using a tetra-polar device (BIA 101 Anniversary; Akern, Florence, Italy). Fat-free mass was determined using the equation published by Schaefer et $\mathrm{al}^{20}$; fat mass was derived accordingly and recoded into tertiles $(<12.8 \% ; 12.8 \%-19.5 \%$; $\geq 19.5 \%$ ).

Maternal height and weight before pregnancy were self-recorded at birth and measured at children's 4-year-old follow-up. Maternal BMI was classified according to the World Health Organization criteria into under/normal weight $\left(\mathrm{BMI}<24.9 \mathrm{~kg} / \mathrm{m}^{2}\right)$ and overweight/obese $\left(\mathrm{BMI} \geq 25.0 \mathrm{~kg} / \mathrm{m}^{2}\right){ }^{21}$ BMI change represents the change in maternal BMI between the prepregnancy period and 4 years after birth.

\section{Children's Eating Behaviors}

Eating behaviors were assessed using the CEBQ, completed by parents at the 7-year-old evaluation ( $94 \%$ were answered by mothers). The original CEBQ ${ }^{22}$ includes 35 items related to eating styles among children, and is answered on a 5-point Likert scale, ranging from $1=$ never to $5=$ always. This questionnaire consists of 8 subdomains related to distinct eating behaviors: satiety responsiveness, slowness in eating, enjoyment of food, food responsiveness, food fussiness, desire for drinks, emotional overeating, and emotional undereating. The CEBQ has demonstrated stability over time and good psychometric properties. ${ }^{22-29} \mathrm{~A}$ version of this questionnaire has been previously validated and adapted to Portuguese children, ${ }^{23}$ but in that version children were aged 3-13 years old, and some items were slightly different from those in the original scale. We opted for retranslating the original CEBQ into Portuguese (Table I; available at www.jpeds.com), resulting in a new Portuguese version CEBQ (P-CEBQ). The psychometric properties of this most recent version (including 7-year-old children) were tested in the present study (Appendix and Table II). A 2-factor solution was identified, explaining $62 \%$ of the total variance: factor 1 -appetite restraint and factor 2 -appetite disinhibition, explaining $35 \%$ and $26 \%$ of the total variance, respectively. The subdomains food fussiness, enjoyment of food, slowness in eating, and satiety responsiveness loaded mostly on factor 1 (loadings: $0.64 ;-0.78 ; 0.74$ and 0.88 ) and the subdomains food responsiveness, emotional overeating, emotional undereating, and desire for drinks loaded mostly on factor 2 (loadings: $0.65,0.82,0.66$, and 0.65 ) (Table II). Thus, appetite restraint was more related to subdomains measuring internal cues of satiety and food fussiness, on which loaded mostly food fussiness, enjoyment of food, slowness in eating, and satiety responsiveness, and appetite disinhibition was more related to subdomains measuring external food cues and emotional responses toward foods, on which loaded mostly food responsiveness, emotional overeating, emotional undereating, and desire for drinks. A short version was proposed for further application in future research. 
Table II. Factor loadings from the principal components analysis for the 35 items of the CEBQ resulting in the original 8-factor structure and the 2-factor final solution of the P-CEBQ used in 7-year-old children $(n=4485)$

\begin{tabular}{|c|c|c|c|c|c|c|c|c|}
\hline \multirow[b]{2}{*}{ \# } & \multirow[b]{2}{*}{ Items } & \multicolumn{5}{|c|}{ Original 8-factor structure } & \multicolumn{2}{|c|}{ Two-factor solution } \\
\hline & & Subdomain & Loading & $\alpha$ & Mean & SD & $\begin{array}{l}\text { Loading } \\
\text { factor } 1\end{array}$ & $\begin{array}{l}\text { Loading } \\
\text { factor } 2\end{array}$ \\
\hline 7 & My child refuses new foods at first. & $\mathrm{FF}$ & -0.80 & 0.84 & 2.95 & 0.76 & 0.64 & 0.19 \\
\hline 10 & My child enjoys tasting new foods. & & 0.87 & & & & & \\
\hline 16 & My child enjoys a wide variety of foods. & & 0.63 & & & & & \\
\hline 24 & My child is difficult to please with meals. & & -0.32 & & & & & \\
\hline 32 & My child is interested in tasting food s/he hasn't tasted before. & & 0.87 & & & & & \\
\hline \multirow[t]{2}{*}{33} & My child decides that s/he doesn't like food, even without tasting it. & & -0.73 & & & & & \\
\hline & Explained variance: & & $10 \%$ & & & & & \\
\hline 12 & My child's always asking for food. & FR & 0.41 & 0.85 & 2.06 & 0.78 & -0.48 & 0.66 \\
\hline 14 & If allowed to, my child would eat too much. & & 0.58 & & & & & \\
\hline 19 & Given the choice, my child would eat most of the time. & & 0.75 & & & & & \\
\hline 28 & Even if my child is full up, s/he finds room to eat his/her favourite food. & & 0.64 & & & & & \\
\hline \multirow[t]{2}{*}{34} & If given the chance, my child would always have food in his/her mouth. & & 0.77 & & & & & \\
\hline & Explained variance: & & $10 \%$ & & & & & \\
\hline 1 & My child loves food. & $\mathrm{EF}$ & 0.67 & 0.83 & 3.03 & 0.79 & -0.78 & 0.32 \\
\hline 5 & My child is interested in food. & & 0.74 & & & & & \\
\hline 20 & My child looks forward to mealtimes. & & 0.30 & & & & & \\
\hline \multirow[t]{2}{*}{22} & My child enjoys eating. & & 0.67 & & & & & \\
\hline & Explained variance: & & $\mathbf{9 \%}$ & & & & & \\
\hline 4 & My child finishes his/her meal very quickly. & SiE & -0.82 & 0.81 & 2.92 & 0.86 & 0.74 & -0.05 \\
\hline 8 & My child eats slowly. & & 0.86 & & & & & \\
\hline 18 & My child takes more than 30 minutes to finish a meal. & & 0.81 & & & & & \\
\hline \multirow[t]{2}{*}{35} & My child eats more and more slowly during the course of a meal. & & 0.63 & & & & & \\
\hline & Explained variance: & & $8 \%$ & & & & & \\
\hline 2 & My child eats more when worried. & EOE & 0.81 & 0.79 & 1.83 & 0.63 & -0.09 & 0.81 \\
\hline 13 & My child eats more when annoyed. & & 0.84 & & & & & \\
\hline 15 & My child eats more when anxious. & & 0.83 & & & & & \\
\hline \multirow[t]{2}{*}{27} & My child eats more when s/he has nothing else to do. & & 0.33 & & & & & \\
\hline & Explained variance: & & $8 \%$ & & & & & \\
\hline 3 & My child has a big appetite. & SR & -0.12 & 0.74 & 2.70 & 0.68 & 0.88 & 0.13 \\
\hline 17 & My child leaves food on his/her plate at the end of a meal. & & 0.69 & & & & & \\
\hline 21 & My child gets full before his/her meal is finished. & & 0.81 & & & & & \\
\hline 26 & My child gets full up easily. & & 0.69 & & & & & \\
\hline \multirow[t]{2}{*}{30} & My child cannot eat a meal if $\mathrm{s} / \mathrm{he}$ has had a snack just before. & & 0.57 & & & & & \\
\hline & Explained variance: & & $8 \%$ & & & & & \\
\hline 9 & My child eats less when s/he is angry. & EUE & 0.78 & 0.76 & 2.46 & 0.76 & 0.47 & 0.66 \\
\hline 11 & My child eats less when $\mathrm{s} / \mathrm{he}$ is tired. & & 0.79 & & & & & \\
\hline 23 & My child eats more when s/he is happy. & & 0.63 & & & & & \\
\hline \multirow[t]{2}{*}{25} & My child eats less when s/he is upset. & & 0.73 & & & & & \\
\hline & Explained variance: & & $7 \%$ & & & & & \\
\hline 6 & My child is always asking for a drink. & $\mathrm{DD}$ & 0.77 & 0.82 & 2.21 & 0.81 & 0.12 & 0.64 \\
\hline 29 & If given the chance, my child would drink continuously throughout the day. & & 0.89 & & & & & \\
\hline \multirow[t]{3}{*}{31} & If given the chance, my child would always be having a drink. & & 0.89 & & & & & \\
\hline & Explained variance: & & $7 \%$ & & & & $35 \%$ & $26 \%$ \\
\hline & Total explained variance: & & $67 \%$ & & & & $62 \%$ & \\
\hline
\end{tabular}

\#, item's position in the original CEBQ; $\alpha$, Cronbach alpha; SiE, slowness in eating

Bold values indicate absolute factor loadings of 0.3 or higher (interpreted as having expressive part on each factor) and the respective explained variances

\section{Statistical Analyses}

Descriptive statistics and generalized linear models ( $\beta$ regression coefficients and the respective $95 \%$ Cis) were performed to estimate the associations. Adjustments for sociodemographics, family structure, and maternal BMI before pregnancy were performed. Further adjustments for smoking during pregnancy, child's BMI z-score, and media screening were also tested, but did not change the magnitude of the associations (results not shown). An interaction of the child's sex in these associations was studied by conducting stratified analysis, but no significant interaction was found; thus, results are reported for both sexes together.

The significance level was set at $P<.05$. SPSS $\mathrm{v} 21.0$ (SPSS Inc, Chicago, Illinois) was used to perform all the statistical analyses, with the exception of the principal component analysis, which was conducted with the software R v 3.0.1 (R Foundation Statistical Computing, Vienna, Austria).

\section{Results}

Table III presents the participants' characteristics $(\mathrm{n}=3562)$ and the appetite restraint and appetite disinhibition scores according to each characteristic (scores could range from 1 to 5). Approximately $51 \%$ were boys, most of them lived in a 2-parent home (89.1\%), and they had no (46.7\%) or 1 sibling $(43.4 \%)$ at 4 years old. At baseline, most mothers were aged between 25 and 35 years (66.3\%), had between 9 and 12 years 
Table III. Descriptive statistics (mean, SD) of the 2 factors identified in the P-CEBQ according to children and maternal characteristics $(n=3562)$

\begin{tabular}{|c|c|c|c|c|c|c|}
\hline & \multirow[b]{2}{*}{$\mathbf{N}$} & \multirow[b]{2}{*}{$\%$} & \multicolumn{2}{|c|}{$\begin{array}{l}\text { Factor } 1 \\
\text { Appetite } \\
\text { restraint }\end{array}$} & \multicolumn{2}{|c|}{$\begin{array}{c}\text { Factor } 2 \\
\text { Appetite } \\
\text { disinhibition }\end{array}$} \\
\hline & & & Mean & SD & Mean & SD \\
\hline \multicolumn{7}{|l|}{ Sociodemographics and family structure } \\
\hline \multicolumn{7}{|l|}{ Maternal age } \\
\hline$<25 y$ & 541 & 15.2 & 2.803 & 0.633 & 2.297 & 0.545 \\
\hline $25-34$ y & 2361 & 66.3 & 2.905 & 0.597 & 2.116 & 0.510 \\
\hline$\geq 35 \mathrm{y}$ & 660 & 18.5 & 2.914 & 0.603 & 2.094 & 0.495 \\
\hline \multicolumn{7}{|l|}{ Maternal education } \\
\hline$<9 y$ & 944 & 26.5 & 2.824 & 0.592 & 2.174 & 0.550 \\
\hline $9-11$ y & 1524 & 42.8 & 2.870 & 0.617 & 2.161 & 0.522 \\
\hline$\geq 12 y$ & 1094 & 30.7 & 2.979 & 0.588 & 2.079 & 0.476 \\
\hline \multicolumn{7}{|l|}{ Family structure at $4 \mathrm{y}$} \\
\hline Two parents & 3174 & 89.1 & 2.899 & 0.602 & 2.127 & 0.508 \\
\hline One parent & 365 & 10.2 & 2.820 & 0.627 & 2.248 & 0.589 \\
\hline Other family structure & 23 & 0.70 & 2.919 & 0.542 & 2.160 & 0.412 \\
\hline \multicolumn{7}{|l|}{ Number of siblings at $4 y$} \\
\hline 0 & 1664 & 46.7 & 2.930 & 0.618 & 2.143 & 0.524 \\
\hline 1 & 1545 & 43.4 & 2.872 & 0.588 & 2.142 & 0.512 \\
\hline$\geq 2$ & 353 & 9.90 & 2.789 & 0.600 & 2.112 & 0.512 \\
\hline \multicolumn{7}{|l|}{ Child's sex } \\
\hline Girls & 1755 & 49.3 & 2.916 & 0.608 & 2.115 & 0.518 \\
\hline Boys & 1807 & 50.7 & 2.867 & 0.601 & 2.164 & 0.516 \\
\hline \multicolumn{7}{|l|}{ Birth characteristics } \\
\hline Type of delivery & & & & & & \\
\hline Vaginal & 2231 & 62.6 & 2.874 & 0.601 & 2.144 & 0.523 \\
\hline Cesarean & 1331 & 37.4 & 2.920 & 0.610 & 2.131 & 0.507 \\
\hline Weight for gestational age & & & & & & \\
\hline Small & 515 & 14.5 & 2.926 & 0.654 & 2.120 & 0.516 \\
\hline Adequate & 2921 & 82.0 & 2.886 & 0.596 & 2.142 & 0.515 \\
\hline Large & 126 & 3.50 & 2.871 & 0.601 & 2.152 & 0.570 \\
\hline Child's characteristics & & & & & & \\
\hline Any breastfeeding duration & & & & & & \\
\hline Never or $<4$ mo & 1437 & 40.3 & 2.870 & 0.616 & 2.159 & 0.539 \\
\hline $4-5 \mathrm{mo}$ & 452 & 12.7 & 2.913 & 0.605 & 2.164 & 0.513 \\
\hline$\geq 6 \mathrm{mo}$ & 1673 & 47.0 & 2.903 & 0.595 & 2.116 & 0.498 \\
\hline Introduction to complementary feeding* & & & & & & \\
\hline$<4 \mathrm{mo}$ & 240 & 6.7 & 2.896 & 0.583 & 2.161 & 0.573 \\
\hline $4-5 \mathrm{mo}$ & 1737 & 48.8 & 2.926 & 0.614 & 2.148 & 0.500 \\
\hline$\geq 6 \mathrm{mo}$ & 721 & 20.2 & 2.890 & 0.574 & 2.150 & 0.490 \\
\hline Complementary feeding — first food* & & & & & & \\
\hline Soup & 981 & 27.5 & 2.847 & 0.602 & 2.171 & 0.530 \\
\hline cereals porridge/fruit/other & 1687 & 47.4 & 2.947 & 0.595 & 2.132 & 0.487 \\
\hline Media screening at $4 \mathrm{y}$ & & & & & & \\
\hline$<120$ min per d & 2540 & 71.3 & 2.884 & 0.603 & 2.118 & 0.512 \\
\hline$\geq 120$ min per $d$ & 1022 & 28.7 & 2.909 & 0.610 & 2.192 & 0.526 \\
\hline Regular physical activity at $4 \mathrm{y}$ & & & & & & \\
\hline No & 1121 & 31.5 & 2.902 & 0.603 & 2.150 & 0.526 \\
\hline Yes & 2441 & 68.5 & 2.886 & 0.605 & 2.135 & 0.513 \\
\hline Child's BMI z-score (WHO) at $4 \mathrm{y}$ & & & & & & \\
\hline Under/normal weight & 2445 & 68.6 & 3.011 & 0.589 & 2.079 & 0.479 \\
\hline Overweight/obese & 1117 & 31.4 & 2.629 & 0.553 & 2.273 & 0.570 \\
\hline Child's WC at 4 y & & & & & & \\
\hline$<50.5 \mathrm{~cm}$ & 1192 & 33.5 & 3.092 & 0.590 & 2.055 & 0.490 \\
\hline $50.5-53.5 \mathrm{~cm}$ & 1201 & 33.7 & 2.915 & 0.563 & 2.112 & 0.503 \\
\hline$\geq 53.6 \mathrm{~cm}$ & 1169 & 32.8 & 2.661 & 0.583 & 2.255 & 0.551 \\
\hline Child's fat $\%$ at $4 y^{*}$ & & & & & & \\
\hline$<12.8 \%$ & 973 & 27.3 & 2.994 & 0.566 & 2.088 & 0.486 \\
\hline $12.8 \%-19.4 \%$ & 1018 & 28.6 & 2.917 & 0.587 & 2.122 & 0.495 \\
\hline$\geq 19.5 \%$ & 1021 & 28.7 & 2.740 & 0.617 & 2.232 & 0.584 \\
\hline Maternal characteristics & & & & & & \\
\hline Maternal BMI before pregnancy & & & & & & \\
\hline Under/normal weight & 2470 & 69.3 & 2.920 & 0.602 & 2.126 & 0.503 \\
\hline Overweight/obese & 1092 & 30.7 & 2.825 & 0.606 & 2.171 & 0.546 \\
\hline Maternal BMI change from before pregnancy to $4 \mathrm{y}$ & & & & & & \\
\hline Maintaining under/normal weight or decreasing & 1838 & 51.6 & 2.861 & 0.607 & 2.151 & 0.534 \\
\hline Maintaining high BMl or increasing & 1724 & 48.4 & 2.923 & 0.601 & 2.127 & 0.499 \\
\hline Smoking during pregnancy & & & & & & \\
\hline Never smoker & 2825 & 79.3 & 2.904 & 0.601 & 2.117 & 0.510 \\
\hline Ever smoker & 737 & 20.7 & 2.839 & 0.616 & 2.226 & 0.536 \\
\hline
\end{tabular}

WHO, World Health Organization.

*These variables do not add to 3562 because of missing data. 
of education $(42.8 \%)$, and were under or of normal weight before pregnancy $(69.3 \%)$.

Associations between children and maternal characteristics and eating behaviors assessed in 7-year-old children are described in Table IV (univariate model) and Table V (multivariate model). In multivariate analysis, older maternal age ( $\geq 35$ vs $<25$ years: $\beta=0.127,95 \%$ CI $0.054,0.201$ ), higher maternal education $(\geq 12$ vs $<9$ years: $\beta=0.109,95 \%$ CI 0.055 , 0.164 ), and having a mother with increased BMI from pregnancy to 4 years after birth or maintaining a high BMI during this period $(\beta=0.043,95 \%$ CI $0.003,0.083)$ were associated with higher appetite disinhibition scores at 7 years old. Children starting complementary feeding with foods other than soup ( $\beta=0.077,95 \%$ CI $0.030,0.124)$ and watching at least 120 minutes of media per day at 4 years old $(\beta=0.048,95 \%$ CI 0.004, 0.092) also showed higher appetite restraint scores at 7 years old. In contrast, girls, children living in a 2-parent family (1-parent vs 2 -parent: $\beta=-0.074,95 \%$ CI -0.140 , $-0.007)$ and with a lower number of siblings ( $\geq 2$ vs 0 : $\beta=-0.152,95 \% \mathrm{CI}-0.224,-0.081)$ were associated with higher appetite restraint scores at 7 years old. Not practicing regular physical activity at 4 years old (yes vs no: $\beta=-0.048$, $95 \%$ CI $-0.091,-0.005)$, lower BMI $(\beta=-0.368,95 \%$ CI -0.410 , -0.327 ), WC (third vs first tertile: $\beta=-0.418,95 \%$ CI -0.46 , -0.371 ), and fat mass percentage at 4 years old (third vs first tertile: $\beta=-0.276,95 \% \mathrm{CI}-0.329,-0.224)$ were also associated with higher appetite restraint scores at 7 years old.

The characteristics significantly associated with higher appetite disinhibition scores at 7 years old were living with 1 parent (1-parent vs 2-parent: $\beta=0.090,95 \%$ CI 0.034, 0.147), being a boy $(\beta=0.051,95 \%$ CI $0.018,0.085)$, and watching at least 120 minutes of media per day $(\beta=0.055,95 \%$ CI 0.018 , $0.093)$. As regards anthropometric characteristics, higher BMI $(\beta=0.183,95 \%$ CI $0.146,0.219)$, WC (third vs first tertile: $\beta=0.188,95 \%$ CI $0.147,0.229)$, and fat mass percentage at 4 years old (third vs first tertile: $\beta=0.160,95 \%$ CI $0.114,0.206$ ) were also associated with higher appetite disinhibition scores at 7 years old. Children of overweight/obese mothers before pregnancy $(\beta=0.046,95 \%$ CI $0.009,0.083)$ and smokers during pregnancy ( $\beta=0.082,95 \%$ CI $0.039,0.124)$ also reported higher appetite disinhibition scores. In turn, higher maternal age $(\geq 35$ vs $<25$ years: $\beta=-0.187,95 \% \mathrm{CI}-0.250,-0.125)$ and education ( $\geq 12$ vs $<9$ years: $\beta=-0.056,95 \%$ CI $-0.102,-0.009$ ) were significantly associated with lower appetite disinhibition scores.

\section{Discussion}

In the present study, higher maternal education and a family with both parents at 4 years old seemed to influence higher appetite restraint, but less appetite disinhibition at 7 years old. More sedentary lifestyles at 4 years old were associated with higher appetite restraint and appetite disinhibition scores later in childhood. An association between high maternal education and low drive to eat in children has been described before ${ }^{12}$ and, more recently, low maternal education and maternal age were associated with overeating in children, ${ }^{10}$ results that corroborate the negative association found in this study between maternal age and education and the appetite disinhibition domain.

Children living in a 1-parent family at 4 years old reported more appetite disinhibition and less appetite restraint at 7 years old than did children living in a 2-parent family. Children with siblings at 4 years old also showed a lower likelihood of developing appetite restraint at 7 years old, an association that was stronger with a higher number of siblings. These findings are in line with previous studies. ${ }^{6,10}$ According to the literature, certain factors in single parenthood ${ }^{30}$ may lead to emotional parenting and feeding practices, with significant impact on children's behavioral development (eg, emotional overeating ${ }^{10,31}$ ). Having a higher number of siblings may also hypothetically decrease parental attention toward each child during mealtimes and pressure to eat, which, in turn, decreases the risk of developing pickiness ${ }^{6,32}$ (characteristic of the appetite restraint domain). Decreased parental attention during mealtimes may also be a consequence of single parenthood. Altogether, the present findings indicate that having a 2-parent family may be positively and negatively associated with appetite restraint and appetite disinhibition, respectively.

Boys reported lower appetite restraint and higher appetite disinhibition scores at 7 years old. Previous studies have reported that girls, relative to boys, have a decreased tendency to overeat, are less interested in food, ${ }^{10,33,34}$ have greater food fussiness, ${ }^{33}$ and have more reported eating difficulties at 2 years of age. ${ }^{12}$

Our results revealed no consistent association between eating behaviors and feeding habits in early infancy. The association with breastfeeding duration was only significant in univariate analysis and showed that infants breastfed for 6 months or longer reported lower appetite disinhibition at 7 years old. Previous literature revealed increased satiety responsiveness in breastfed children, ${ }^{13,14,35}$ suggesting that breastfeeding may allow infants to learn how to regulate their appetite and respond to internal cues of satiety ${ }^{13}$ and, thus, protect them from susceptibility to external intake cues (maternal judgement of timing, volumes, and energy density at each feeding).

The type of food first consumed (but not the timing) at complementary feeding significantly predicted eating behaviors at 7 years old. Children who first consumed cereal porridge and fruit reported more appetite restraint at 7 years old than did children whose first food was vegetable soup. Recommendations ${ }^{36}$ are increasing toward the prioritization of vegetable soup because of the higher energy density and sweetness of cereal porridge. Infants have an innate preference for sweet tastes, ${ }^{37}$ and, thus, the introduction of these more pleasurable foods may be easily accepted, but compromise the development of self-regulation intake mechanisms, ${ }^{38}$ thereby supporting the positive association between cereal porridge and other sweet foods and the appetite restraint domain found in our study. The absence of repeated exposure to vegetables and unsweetened fruits early in life may even induce a low capacity to taste different flavours later in life, ${ }^{38}$ consequently promoting picky eating.

More sedentary behaviors (no regular practice of physical activity and a high media screening time) at 4 years old were 


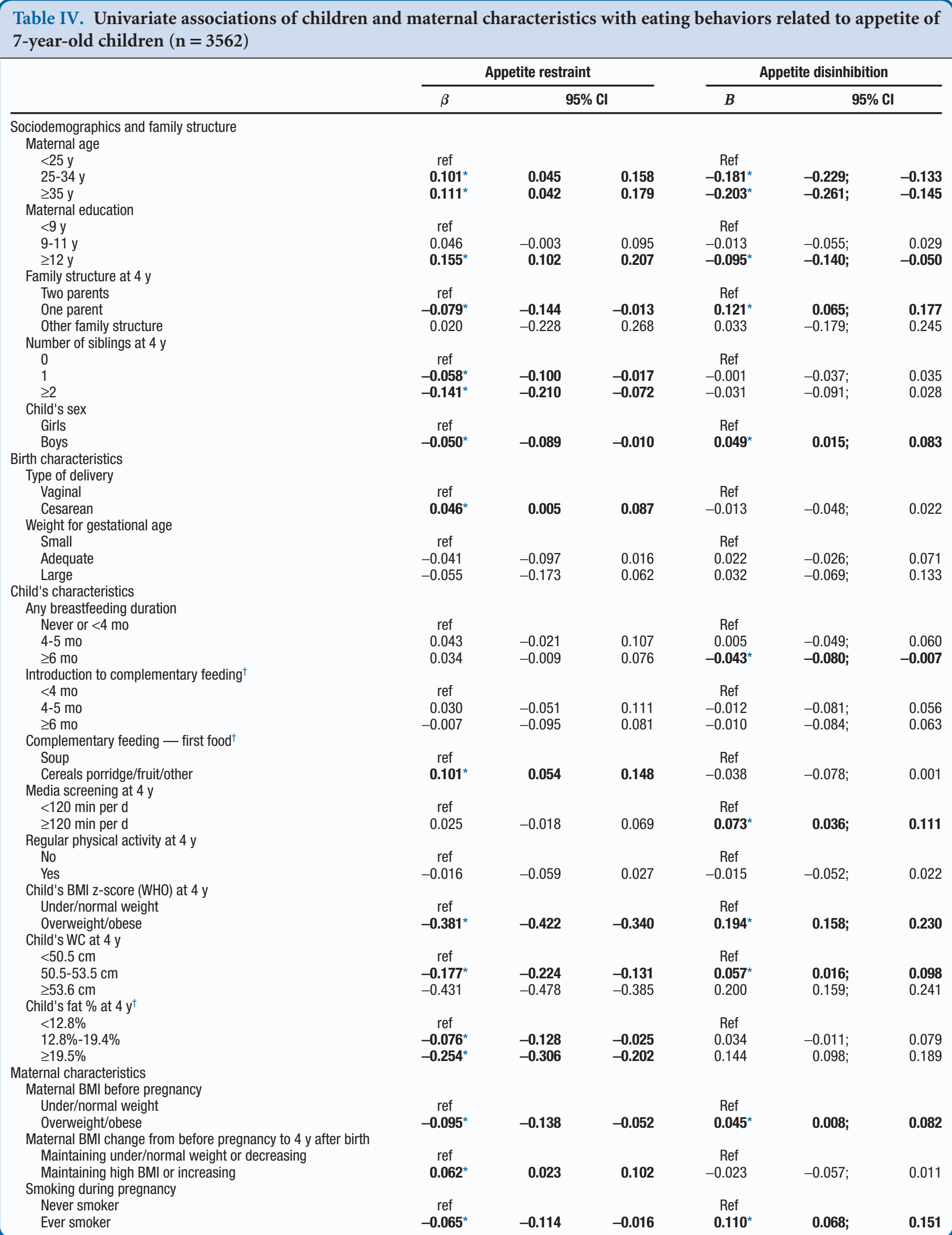

*Significant associations are in bold type and marked with an asterisk. †For these variables, sample sizes are lower (as described in Table I). 


\begin{tabular}{|c|c|c|c|c|c|c|}
\hline \multirow{2}{*}{ Sociodemographics and family structure } & \multicolumn{3}{|c|}{ Appetite restraint } & \multicolumn{3}{|c|}{ Appetite disinhibition } \\
\hline & $\beta^{\star}$ & \multicolumn{2}{|c|}{$95 \% \mathrm{Cl}$} & $\beta^{\star}$ & \multicolumn{2}{|c|}{$95 \% \mathrm{Cl}$} \\
\hline \multicolumn{7}{|l|}{ Maternal age } \\
\hline$<25$ y & ref & & & Ref & & \\
\hline $25-34$ y & $0.083^{\star}$ & 0.024 & 0.142 & $-0.161^{\star}$ & -0.212 & -0.110 \\
\hline$\geq 35$ y & $0.127^{\star}$ & 0.054 & 0.201 & $-0.187^{\star}$ & -0.250 & -0.125 \\
\hline \multicolumn{7}{|l|}{ Maternal education } \\
\hline$<9 y$ & ref & & & Ref & & \\
\hline $9-11 y$ & 0.028 & -0.021 & 0.078 & -0.017 & -0.059 & 0.025 \\
\hline$\geq 12 y$ & $0.109^{*}$ & 0.055 & 0.164 & $-0.056^{\star}$ & -0.102 & -0.009 \\
\hline \multicolumn{7}{|l|}{ Family structure at $4 \mathrm{y}$} \\
\hline Two parents & ref & & & ref & & \\
\hline One parent & $-0.074^{\star}$ & -0.140 & -0.007 & $0.090^{\star}$ & 0.034 & 0.147 \\
\hline Other family structure & 0.007 & -0.239 & 0.253 & -0.001 & -0.211 & 0.209 \\
\hline \multicolumn{7}{|l|}{ Number of siblings at $4 \mathrm{y}$} \\
\hline 0 & ref & & & ref & & \\
\hline 1 & $-0.077^{\star}$ & -0.120 & -0.040 & 0.028 & -0.009 & 0.064 \\
\hline$\geq 2$ & $-0.152^{\star}$ & -0.224 & -0.081 & -0.006 & -0.067 & 0.055 \\
\hline Child's sex & & & & & & \\
\hline Girls & ref & & & ref & & \\
\hline Boys & $-0.053^{\star}$ & -0.092 & -0.014 & $0.051^{*}$ & 0.018 & 0.085 \\
\hline Birth characteristics & & & & & & \\
\hline Type of delivery & & & & & & \\
\hline Vaginal & ref & & & ref & & \\
\hline Cesarean & 0.028 & -0.013 & 0.069 & 0.002 & -0.033 & 0.037 \\
\hline Weight for gestational age & & & & & & \\
\hline Small & ref & & & ref & & \\
\hline Adequate & -0.039 & -0.096 & 0.017 & 0.029 & -0.019 & 0.077 \\
\hline Large & -0.043 & -0.160 & 0.074 & 0.042 & -0.058 & 0.142 \\
\hline Child's characteristics & & & & & & \\
\hline Any breastfeeding duration & & & & & & \\
\hline Never or $<4$ mo & ref & & & ref & & \\
\hline $4-5 \mathrm{mo}$ & 0.029 & -0.035 & 0.092 & 0.021 & -0.033 & 0.075 \\
\hline$\geq 6 \mathrm{mo}$ & 0.021 & -0.021 & 0.064 & -0.025 & -0.061 & 0.012 \\
\hline Introduction to complementary feeding ${ }^{\dagger}$ & & & & & & \\
\hline$<4 \mathrm{mo}$ & ref & & & ref & & \\
\hline $4-5 \mathrm{mo}$ & -0.004 & -0.084 & 0.077 & 0.008 & -0.059 & 0.076 \\
\hline$\geq 6 \mathrm{mo}$ & -0.026 & -0.112 & 0.061 & 0.012 & -0.067 & 0.078 \\
\hline Complementary feeding — first food ${ }^{\dagger}$ & & & & & & \\
\hline Soup & ref & & & ref & & \\
\hline Cereals porridge/fruit/other & $0.077^{\star}$ & 0.030 & 0.124 & -0.018 & -0.057 & 0.022 \\
\hline Media screening at $4 \mathrm{y}$ & & & & & & \\
\hline$<120$ min per d & ref & & & Ref & & \\
\hline$\geq 120$ min per $d$ & $0.048^{\star}$ & 0.004 & 0.092 & $0.055^{\star}$ & 0.018 & 0.093 \\
\hline Regular physical activity at $4 \mathrm{y}$ & & & & & & \\
\hline  & ref & & & Ref & & \\
\hline Yes & $-0.048^{\star}$ & -0.091 & -0.005 & 0.005 & -0.032 & 0.042 \\
\hline Child's BMI z-score (WHO) at $4 \mathrm{y}$ & & & & & & \\
\hline Under/normal weight & ref & & & Ref & & \\
\hline Overweight/obese & $-0.368^{\star}$ & -0.410 & -0.327 & $0.183^{\star}$ & 0.146 & 0.219 \\
\hline Child's WC at $4 \mathrm{y}$ & & & & & & \\
\hline$<50.5 \mathrm{~cm}$ & ref & & & Ref & & \\
\hline $50.5-53.5 \mathrm{~cm}$ & $-0.171^{*}$ & -0.217 & -0.125 & $0.052^{\star}$ & 0.011 & 0.092 \\
\hline$\geq 53.6 \mathrm{~cm}$ & $-0.418^{\star}$ & -0.465 & -0.371 & $0.188^{\star}$ & 0.147 & 0.229 \\
\hline Child's fat $\%$ at $4 \mathrm{y}^{\dagger}$ & & & & & & \\
\hline$<12.8 \%$ & ref & & & Ref & & \\
\hline $12.8 \%-19.4 \%$ & $-0.092^{\star}$ & -0.144 & -0.041 & $0.047^{\star}$ & 0.002 & 0.092 \\
\hline$\geq 19.5 \%$ & $-0.276^{\star}$ & -0.329 & -0.224 & $0.160^{\star}$ & 0.114 & 0.206 \\
\hline Maternal characteristics & & & & & & \\
\hline Maternal BMI before pregnancy & & & & & & \\
\hline Under/normal weight & ref & & & Ref & & \\
\hline Overweight/obese & $-0.077^{\star}$ & -0.12 & -0.033 & $0.046^{\star}$ & 0.009; & 0.083 \\
\hline Maternal BMI change from before pregnancy to $4 \mathrm{y}$ & & & & & & \\
\hline Maintaining under/normal weight or decreasing & ref & & & Ref & & \\
\hline Maintaining high BMI or increasing & $0.043^{\star}$ & 0.003 & 0.083 & -0.020 & -0.055 & 0.014 \\
\hline Smoking during pregnancy & & & & & & \\
\hline Never smoker & ref & & & Ref & & \\
\hline Ever smoker & -0.045 & -0.095 & 0.004 & $0.082^{\star}$ & 0.039; & 0.124 \\
\hline
\end{tabular}

*Significant associations are in bold type and marked with an asterisk.

†For these variables, sample sizes are lower (as described in Table I).

$\beta$ adjusted for maternal age, education, and BMI before birth, family structure, and number of siblings at 4 years old and child's sex. 
associated with higher appetite restraint and appetite disinhibition at 7 years old. Accordingly, emotional and external eating have already been associated with increased screen time, ${ }^{39}$ which could be explained by an association between screen time and less attentive eating. ${ }^{40,41}$

Overweight, obese children and those with higher adiposity at 4 years old reported low appetite restraint and high appetite disinhibition at 7 years old. Obese children have been characterized by lower responsiveness to internal satiety cues ${ }^{42,43}$ and food fussiness, ${ }^{5}$ eating faster during the course of a meal, ${ }^{3}$ presenting more food enjoyment, ${ }^{4,5}$ sensitiveness to external food cues, ${ }^{5,23,44}$ and emotional states, ${ }^{5}$ and higher desire for drinks $^{5}$ than healthy-weight children. Consistent findings were reported in the previous Portuguese study conducted with children and adolescents. ${ }^{23}$ Most of these studies have, however, a cross-sectional design and do not clarify the actual direction of these associations. Our findings add weight status/ adiposity in preschool children as a possible determinant of appetite later in childhood.

Children of overweight mothers also had lower appetite restraint and higher appetite disinhibition at 7 years old, and children whose mothers smoked during pregnancy reported high appetite disinhibition in later childhood, which may indicate that unhealthy maternal lifestyles are predictors of problematic eating behaviors in children. Research has shown a tendency for overweight mothers to present individual eating concerns to develop more restrictive feeding styles when they perceive their children as overweight or at risk of developing weight problems. ${ }^{37,38,45-47}$ Contrasting results were found when we studied the maternal BMI before pregnancy. These conflicting findings may represent a wider link between parental weight, self-image, concerns about children's weight and feeding practices, and children's eating behaviors. Further investigation into the characteristics referred to should bring some insight into the present findings.

Smoking during pregnancy has been associated with higher overweight risk in children ${ }^{48}$ which has been suggested to be to be due to, and explained, in part, by, the intrauterine exposure to inhaled smoke products, which decreases appetitive learning of responsiveness to internal cues, by acting on neurotransmitters associated with reward. ${ }^{49}$ Behavioral associations linking parental smoking habits with unhealthy lifestyles, particularly dietary habits, are also important to consider. ${ }^{48}$

This study has some limitations. Self-administered questionnaires often present low response rates, and a relatively high number of children had incomplete or no information on the CEBQ. Compared with the entire cohort, the mothers in the current sample were slightly older and more educated. Selective response bias, however, is not of concern as these differences were not relevant. The Cohen effect size values were lower than 0.35 (low magnitude), ${ }^{50}$ suggesting that differences were mostly because of the large sample size rather than to large differences between participants' characteristics. In addition, we were able to recover missing data in cases with more than $50 \%$ of the items completed. Nevertheless, we hope to overcome this barrier in future cohort evaluations by introducing the shorter version defined in this study. In addition, the assessment of eating behaviors was based on parental report, which may introduce measurement error because of inherent subjectivity. Social desirability should not be of concern in this study because the CEBQ has shown good correspondence with objective measures. ${ }^{26}$ Future research should clarify whether an acceptable level of restraint could have a beneficial effect on appetite. Finally, some variables were asked about at the 4- and 7-year-old follow-up assessments and measured retrospectively, depending on parents' memory, introducing a possible recall bias.

These results provide relevant insights into some early influences of appetite-related eating behaviors in children, which can be useful in future research and in support of the development of prevention guidelines and educational strategies involving families aimed at improving healthy eating behaviors and reducing childhood obesity.

Submitted for publication Feb 24, 2016; last revision received Aug 3, 2016; accepted Sep 7, 2016

Reprint requests: Andreia Oliveira, PhD, Institute of Public Health, Universidade do Porto, Rua das Taipas, $n^{\circ} 135$, 4050-600 Porto, Portugal. E-mail: acmatos@med.up.pt

\section{References}

1. Mennella JA, Beauchamp GK. Flavor experiences during formula feeding are related to preferences during childhood. Early Hum Dev 2002;68:7182.

2. Birch LL, Fisher JO. Development of eating behaviors among children and adolescents. Pediatrics 1998;101:539-49.

3. Barkeling B, Ekman S, Rossner S. Eating behaviour in obese and normal weight 11-year-old children. Int J Obes Relat Metab Disord 1992;16:35560.

4. Carnell S, Wardle J. Appetite and adiposity in children: evidence for a behavioral susceptibility theory of obesity. Am J Clin Nutr 2008;88:229.

5. Webber L, Hill C, Saxton J, Van Jaarsveld CH, Wardle J. Eating behaviour and weight in children. Int J Obes 2009;33:21-8.

6. Hafstad GS, Abebe DS, Torgersen L, von Soest T. Picky eating in preschool children: the predictive role of the child's temperament and mother's negative affectivity. Eat Behav 2013;14:274-7. doi:10.1016/ j.eatbeh.2013.04.001.

7. Carnell S, Wardle J. Appetitive traits and child obesity: measurement, origins and implications for intervention. Proc Nutr Soc 2008;67:343-55.

8. Carnell S, Wardle J. Appetitive traits in children. New evidence for associations with weight and a common, obesity-associated genetic variant. Appetite 2009;53:260-3.

9. Carruth BR, Ziegler PJ, Gordon A, Barr SI. Prevalence of picky eaters among infants and toddlers and their caregivers' decisions about offering a new food. J Am Diet Assoc 2004;104:s57-64.

10. Dubois L, Farmer A, Girard M, Peterson K, Tatone-Tokuda F. Problem eating behaviors related to social factors and body weight in preschool children: a longitudinal study. Int J Behav Nutr Phys Act 2007;4: 9.

11. Oliveira A, de Lauzon-Guillain B, Jones L, Emmett P, Moreira P, Ramos E, et al. Birth weight and eating behaviors of young children. J Pediatr 2015; 166:59-65, e3.

12. Migraine A, Nicklaus S, Parnet P, Lange C, Monnery-Patris S, Des Robert C, et al. Effect of preterm birth and birth weight on eating behavior at 2 y of age. Am J Clin Nutr 2013;97:1270-7. doi:10.3945/ajcn.112.051151.

13. Brown A, Lee M. Breastfeeding during the first year promotes satiety responsiveness in children aged 18-24 months. Pediatr Obes 2012;7:38290. doi:10.1111/j.2047-6310.2012.00071. 
14. Reyes M, Hoyos V, Martinez SM, Lozoff B, Castillo M, Burrows R, et al. Satiety responsiveness and eating behavior among Chilean adolescents and the role of breastfeeding. Int J Obes (Lond) 2014;38:552-7. doi:10.1038/ ijo.2013.191.

15. Jacobi C, Agras WS, Bryson S, Hammer LD. Behavioral validation, precursors, and concomitants of picky eating in childhood. J Am Acad Child Adolesc Psychiatry 2003;42:76-84.

16. Sullivan SA, Birch LL. Infant dietary experience and acceptance of solid foods. Pediatrics 1994;93:271-7.

17. Larsen PS, Kamper-Jørgensen M, Adamson A, Barros H, Bonde JP, Brescianini S, et al. Pregnancy and birth cohort resources in Europe: a large opportunity for aetiological child health research. Paediatr Perinat Epidemiol 2013;27:393-414. doi:10.1111/ppe.12060.

18. Kramer MS, Platt RW, Wen SW, Joseph KS, Allen A, Abrahamowicz M, et al. A new and improved population-based Canadian reference for birth weight for gestational age. Pediatrics 2001;108:E35.

19. WHO, Child WHO. Growth Standards based on length/height, weight and age. Acta Paediatr Suppl 2006;450:76-85.

20. Schaefer F, Georgi M, Zieger A, Scharer K. Usefulness of Bioelectric Impedance and Skinfold Measurements in Predicting Fat-Free Mass Derived from Total Body Potassium in Children. Pediatr Res 1994;35:61724.

21. Clinical guidelines on the identification, evaluation, and treatment of overweight and obesity in adults: executive summary. Expert Panel on the Identification, Evaluation, and Treatment of Overweight in Adults. Am J Clin Nutr 1998;68:899-917.

22. Wardle J, Guthrie CA, Sanderson S, Rapoport L. Development of the children's eating behaviour questionnaire. J Child Psychol Psychiatry 2001;42:963-70.

23. Viana V, Sinde S, Saxton JC. Children's Eating Behaviour Questionnaire: associations with BMI in Portuguese children. Br J Nutr 2008; 100:445-50.

24. Sleddens E, Kremers S, Thijs C. The Children's Eating Behaviour Questionnaire: factorial validity and association with Body Mass Index in Dutch children aged 6-7. Int J Behav Nutr Phys Act 2008;5:49.

25. Svensson V, Lundborg L, Cao Y, Nowicka P, Marcus C, Sobko T. Obesity related eating behaviour patterns in Swedish preschool children and association with age, gender, relative weight and parental weight - factorial validation of the Children's Eating Behaviour Questionnaire. Int J Behav Nutr Phys Act 2011;8:134.

26. Carnell S, Wardle J. Measuring behavioural susceptibility to obesity: validation of the child eating behaviour questionnaire. Appetite 2007;48:104-13.

27. Ashcroft J, Semmler C, Carnell S, van Jaarsveld CHM, Wardle J. Continuity and stability of eating behaviour traits in children. Eur J Clin Nutr 2008;62:985-90.

28. Farrow C, Blissett J. Stability and continuity of parentally reported child eating behaviours and feeding practices from 2 to 5 years of age. Appetite 2012;58:151-6. doi:10.1016/j.appet.2011.09.005.

29. Parkinson KN, Drewett RF, Le Couteur AS, Adamson AJ. Do maternal ratings of appetite in infants predict later Child Eating Behaviour Questionnaire scores and body mass index? Appetite 2010;54:18690.

30. Ryan RM, Claessens A. Associations between family structure changes and children's behavior problems: the moderating effects of timing and marital birth. Dev Psychol 2013;49:1219-31.
31. Braden A, Rhee K, Peterson CB, Rydell SA, Zucker N, Boutelle K. Associations between child emotional eating and general parenting style, feeding practices, and parent psychopathology. Appetite 2014;80:35-40.

32. Galloway AT, Fiorito L, Lee Y, Birch LL. Parental pressure, dietary patterns, and weight status among girls who are "picky eaters". J Am Diet Assoc 2005;105:541-8.

33. Cao Y-T, Svensson V, Marcus C, Zhang J, Zhang J-D, Sobko T. Eating behaviour patterns in Chinese children aged 12-18 months and association with relative weight - factorial validation of the Children's Eating Behaviour Questionnaire. Int J Behav Nutr Phys Act 2012;9:5. doi:10.1186/ 479-5868-9-5.

34. Santos J, Ho-Urriola J, Gonzalez A, Smalley S, Dominguez-Vasquez P, Cataldo R, et al. Association between eating behavior scores and obesity in Chilean children. Nutr J 2011;10:108.

35. Disantis KI, Collins BN, Fisher JO, Davey A. Do infants fed directly from the breast have improved appetite regulation and slower growth during early childhood compared with infants fed from a bottle? Int J Behav Nutr Phys Act 2011;8:89. doi:10.1186/479-5868-8-89.

36. Guerra A, Rêgo C, Silva D, Ferreira GC, Mansilha H, Antunes H, et al. Alimentação e nutrição do lactente. 2012.

37. Birch LL. Development of food preferences. Annu Rev Nutr 1999;19:4162.

38. Savage JS, Fisher JO, Birch LL. Parental influence on eating behavior: conception to adolescence. J Law Med Ethics 2007;35:22-34.

39. van Strien T, Oosterveld P. The children's DEBQ for assessment of restrained, emotional, and external eating in 7- to 12-year-old children. Int J Eat Disord 2008;41:72-81.

40. Brown KW, Ryan RM. The benefits of being present: mindfulness and its role in psychological well-being. J Pers Soc Psychol 2003;84:822-48.

41. Ray C, Roos E. Family characteristics predicting favourable changes in 10 and 11-year-old children's lifestyle-related health behaviours during an 18-month follow-up. Appetite 2012;58:326-32.

42. Fisher JO, Birch LL. Eating in the absence of hunger and overweight in girls from 5 to 7 y of age. Am J Clin Nutr 2002;76:226-31.

43. Fisher JO, Cai G, Jaramillo SJ, Cole SA, Comuzzie AG, Butte NF. Heritability of hyperphagic eating behavior and appetite-related hormones among Hispanic children. Obesity (Silver Spring) 2007;15:1484-95.

44. Jansen A, Theunissen N, Slechten K, Nederkoorn C, Boon B, Mulkens S, et al. Overweight children overeat after exposure to food cues. Eat Behav 2003;4:197-209.

45. Scaglioni S, Salvioni M, Galimberti C. Influence of parental attitudes in the development of children eating behaviour. Br J Nutr 2008;99:S22-5. doi:10.1017/S0007114508892471.

46. Shunk JA, Birch LL. Girls at risk for overweight at age 5 are at risk for dietary restraint, disinhibited overeating, weight concerns, and greater weight gain from 5 to 9 years. J Am Diet Assoc 2004;104:1120-6.

47. Birch LL, Doub AE. Learning to eat: birth to age 2 y. Am J Clin Nutr 2014;99:723S-8S. doi:10.3945/ajcn.113.069047.

48. Oken E, Levitan EB, Gillman MW. Maternal smoking during pregnancy and child overweight: systematic review and meta-analysis. Int J Obes 2007;32:201-10.

49. von Kries R, Toschke AM, Koletzko B, Slikker W. Maternal Smoking during Pregnancy and Childhood Obesity. Am J Epidemiol 2002;156:954-61.

50. Husted JA, Cook RJ, Farewell VT, Gladman DD. Methods for assessing responsiveness: a critical review and recommendations. J Clin Epidemiol 2000;53:459-68. 


\section{Appendix}

\section{Determination of P-CEBQ Psychometric Properties} Factor Analysis and Internal Consistency. Principal component analyses (PCAs) were performed twice in this study. A PCA with Oblimin rotation was first performed on all items of the CEBQ to understand if the original factor structure (with 8 factors, representing 8 subdomains) would be replicated in the present sample. An 8-factor structure that explained $67 \%$ of the total variance was identified (Table II). Most of the items loaded on the expected subdomains, as previously reported. ${ }^{1-5}$ A second PCA was later conducted on the emerged subdomains, and the scree plot supported an alternative 2-factor solution (representing 2 domains), explaining 62\% of the total variance, with factor 1 and factor 2 explaining $35 \%$ and $26 \%$ of the total variance, respectively. The items with absolute factor loading of 0.3 or higher were interpreted as having meaningful part on each factor. The subdomains food fussiness, enjoyment of food, slowness in eating, and satiety responsiveness loaded mostly on factor 1 (loadings: $0.64 ;-0.78 ; 0.74$ and 0.88 ) and the subdomains food responsiveness, emotional overeating, emotional undereating, and desire for drinks loaded mostly on factor 2 (loadings: $0.65 ; 0.82 ; 0.66$ and 0.65 ) (Table II). Factor 1 was subjectively named "appetite restraint" and factor 2 "appetite disinhibition."

To assess the internal consistency of the CEBQ scale, Cronbach alpha coefficients for each subdomain and Pearson correlation coefficients between subdomains were calculated. Good internal consistency was defined by a Cronbach alpha of 0.7 or higher and a Pearson correlation of 0.4 or higher. Both PCAs revealed good internal consistency; in the first, Cronbach alpha for the different subdomains ranged from 0.74 to 0.85 and in the second, Cronbach alpha was 0.77 for "appetite restraint" and 0.64 for "appetite disinhibition." The correlation between both domains was weak ( rho $=-0.013$ ), indicating that they are independent from each other, representing very different aspects of eating behaviors.

For each subdomain defined through the PCA, descriptive statistics (mean, SD) were calculated. A higher score in a subdomain indicated a higher presence of the eating behavior (Table II). The average score in appetite restraint was 2.88, $\mathrm{SD}=0.61$ and in appetite disinhibition was $2.15, \mathrm{SD}=0.52$. In accordance with the original CEBQ, the scores of 5 items were reversed because of opposite phrasing (Appendix). In the 2 -factor solution, only the score of the enjoyment of food subdomain was reversed (factor 1).

Construct Validity. Construct validity was assessed considering theoretical hypotheses based on previously described literature: as higher the children's BMI, higher the emotional eating and responsiveness to external cues ${ }^{6-8}$ and lower the food fussiness $^{1,2}$ and responsiveness to internal satiety cues. A similar relation was hypothesized with higher maternal BMI. ${ }^{9}$ It was performed on the 2 factors emerged in the second PCA. The mean values of each factor by children's BMI and maternal prepregnancy BMI were compared using 1-way ANOVA. As expected, children's BMI was negatively and significantly associated with appetite restraint and positively associated with appetite disinhibition; overweight/obese children scored significantly lower on appetite restraint $($ mean $=2.60[\mathrm{SD}=0.55]$ vs 3.05 [0.58]; $P<.001)$ and higher on appetite disinhibition (2.07 [0.48] vs 2.28 [0.57]; $P<.001)$. Regarding maternal BMI, children with overweight/obese mothers also scored significantly lower on appetite restraint $(2.82[0.61]$ vs 2.91 [0.60]; $P<.001)$ and significantly higher on appetite disinhibition $(2.17$ [0.55] vs $2.14[0.51] ; P=.026)$. The referred associations were consistent with the theoretical hypothesis, supporting the construct validity of the P-CEBQ scale.

Reliability. Reliability was tested by analyzing the agreement between the subdomains in 2 time moments (CEBQ was answered again 35 days apart) using 2-way random intraclass correlation (ICC). ${ }^{10}$ ICC varies between 0 and 1 , with higher values representing higher reliability. The mean ICC of the P-CEBQ ranged from 0.51 (desire for drinks subdomain) to 0.85 (food fussiness subdomain). Most subdomains presented good reliability (ICC $\geq 0.7$ ), except desire for drinks $(\mathrm{ICC}=0.51)$ and emotional undereating $(\mathrm{ICC}=0.58)$. Overall, the P-CEBQ presented a good reliability in this Portuguese sample of 7 -year-olds (mean ICC $=0.73$ ).

Short Version of the Scale. A Spearman-Brown prediction formula was conducted to estimate the hypothetical Cronbach alpha in a new version of the CEBQ scale with fewer items. It was set to retain in each subdomain only the necessary number of items to achieve a Cronbach alpha of at least 0.7. A total of 20 items (in replacement of the original 35 items) were retained in this short-version of the P-CEBQ. Our analysis showed that only 2 items would be enough to represent the subdomains enjoyment of food, food responsiveness, desire for drinks and slowness in eating, and 3 items would be enough to represent the subdomains emotional undereating, emotional overeating, satiety responsiveness, and food responsiveness. In this version, the Cronbach alpha ranged from 0.68 to 0.72 , showing a good internal consistency. As there is no consensus on a method to define which items to retain (ie, to include in the shortversion), we, therefore, suggest that this decision should be taken by each research group, concerning 2 main criteria: loadings and pertinence of the items in each subdomain.

\section{References}

1. Wardle J, Guthrie CA, Sanderson S, Rapoport L. Development of the children's eating behaviour questionnaire. J Child Psychol Psychiatry 2001;42:963-70. doi:10.1111/1469-7610.00792.

2. Viana V, Sinde S, Saxton JC. Children's Eating Behaviour Questionnaire: associations with BMI in Portuguese children. Br J Nutr 2008;100:445-50. doi:10.1017/S0007114508894391.

3. Sleddens E, Kremers S, Thijs C. The Children's Eating Behaviour Questionnaire: factorial validity and association with Body Mass Index in Dutch children aged 6-7. Int J Behav Nutr Phys Act 2008;5:49.

4. Svensson V, Lundborg L, Cao Y, Nowicka P, Marcus C, Sobko T. Obesity related eating behaviour patterns in Swedish preschool children and association with age, gender, relative weight and parental weight - factorial validation of the Children's Eating Behaviour Questionnaire. Int J Behav Nutr Phys Act 2011;8:134. 
5. Santos J, Ho-Urriola J, Gonzalez A, Smalley S, Dominguez-Vasquez P, Cataldo R, et al. Association between eating behavior scores and obesity in Chilean children. Nutr J 2011;10:108.

6. Fisher JO, Cai G, Jaramillo SJ, Cole SA, Comuzzie AG, Butte NF. Heritability of hyperphagic eating behavior and appetite-related hormones among Hispanic children. Obesity (Silver Spring) 2007;15:1484-95.

7. Gregory J, Paxton S, Brozovic A. Maternal feeding practices, child eating behaviour and body mass index in preschool-aged children: a prospective analysis. Int J Behav Nutr Phys Act 2010;7:55.
8. Joyce JL, Zimmer-Gembeck MJ. Parent feeding restriction and child weight. The mediating role of child disinhibited eating and the moderating role of the parenting context. Appetite 2009;52:726-34. doi:10.1016/ j.appet.2009.03.015.

9. Dubois L, Farmer A, Girard M, Peterson K, Tatone-Tokuda F. Problem eating behaviors related to social factors and body weight in preschool children: a longitudinal study. Int J Behav Nutr Phys Act 2007;4:9.

10. McGraw KO, Wong S. "Forming inferences about some intraclass correlations coefficients": correction. 1996

Table I. Correspondence between the original items of the CEBQ and the current P-CEBQ used in 7-year-old children

Original items of the CEBQ

Items of the P-CEBQ

CEBQ

1. My child loves food.

2. My child eats more when worried.

3. My child has a big appetite. $R$

4. My child finishes his/her meal very quickly. $R$

5. My child is interested in food.

6. My child is always asking for a drink.

7. My child refuses new foods at first.

8. My child eats slowly.

9. My child eats less when s/he is angry.

10. My child enjoys tasting new foods. R

11. My child eats less when $\mathrm{s} / \mathrm{he}$ is tired.

12. My child's always asking for food.

13. My child eats more when annoyed.

14. If allowed to, my child would eat too much.

15. My child eats more when anxious.

16. My child enjoys a wide variety of foods. $R$

17. My child leaves food on his/her plate at the end of a meal.

18. My child takes more than 30 minutes to finish a meal.

19. Given the choice, my child would eat most of the time.

20. My child looks forward to mealtimes.

21. My child gets full before his/her meal is finished.

22. My child enjoys eating.

23. My child eats more when s/he is happy.

24. My child is difficult to please with meals.

25. My child eats less when s/he is upset.

26. My child gets full up easily.

27. My child eats more when $\mathrm{s} / \mathrm{he}$ has nothing else to do.

28. Even if my child is full up, s/he finds room to eat his/her

favourite food.

29. If given the chance, my child would drink continuously

throughout the day.

30. My child cannot eat a meal if s/he has had a snack just before.

31. If given the chance, my child would always be having a drink.

32. My child is interested in tasting food $\mathrm{s} /$ he hasn't tasted before. $\mathrm{R}$

33. My child decides that s/he doesn't like food, even without tasting it.

34. If given the chance, my child would always have food in

his/her mouth.

35. My child eats more and more slowly during the course of a meal.

1. 0 meu filho(a) adora comida.

2. 0 meu filho(a) come mais quando anda preocupado(a).

3. 0 meu filho(a) tem muito apetite. $R$

4. 0 meu filho(a) termina as refeições muito rapidamente. $R$

5. 0 meu filho(a) interessa-se por comida.

6. 0 meu filho(a) anda sempre a pedir para beber.

7. Perante novos alimentos o meu filho(a) começa por recusá-los.

8. 0 meu filho(a) come lentamente.

9. 0 meu filho(a) come menos quando está zangado(a).

10. 0 meu filho(a) gosta de experimentar novos alimentos. $R$

11. 0 meu filho(a) come menos quando está cansado(a).

12. 0 meu filho(a) está sempre a pedir comida.

13. 0 meu filho(a) come mais quando está aborrecido(a).

14. Se 0 deixassem o meu filho(a) comeria demais.

15. 0 meu filho(a) come mais quando está ansioso(a)

16. 0 meu filho(a) gosta de uma grande variedade de alimentos. $R$

17. 0 meu filho(a) deixa comida no prato no fim das refeições.

18. 0 meu filho(a) demora mais que 30 minutos para terminar uma refeição.

19. Se tivesse oportunidade o meu filho(a) passaria a maior parte do tempo a comer.

20. 0 meu filho(a) está sempre à espera da hora das refeições

21. 0 meu filho(a) fica cheio/saciado(a) antes de terminar a refeição.

22. 0 meu filho(a) tem prazer em comer.

23. 0 meu filho(a) come mais quando está feliz.

24. 0 meu filho(a) é difícil de agradar com as refeições.

25. 0 meu filho(a) come menos quando anda chateado(a).

26. 0 meu filho(a) fica cheio/saciado(a) muito facilmente.

27. 0 meu filho(a) come mais quando não tem nada para fazer.

28. Mesmo se já está cheio o meu filho(a) arranja espaço para comer um alimento preferido.

29. Se tivesse oportunidade o meu filho(a) passaria o dia a beber continuamente.

30. 0 meu filho(a) é incapaz de comer a refeição se antes tiver comido alguma coisa.

31. Se tivesse oportunidade o meu filho(a) passaria a maior parte do tempo a beber.

32. 0 meu filho(a) interessa-se por experimentar alimentos que nunca provou antes. $\mathrm{R}$

33. 0 meu filho(a) decide que não gosta de um alimento mesmo que nunca 0 tenha provado.

34. Se tivesse oportunidade o meu filho(a) estaria sempre com comida na boca.

35. 0 meu filho(a) come cada vez mais devagar ao longo da refeição.

subdomains

EF

EOE

SR

SiE

EF

DD

FF

SiE

EUE

FF

EUE

FR

EOE

FR

EOE

FF

SR

SiE

FR

EF

SR

EF

EUE

FF

EUE

SR

EOE

FR

DD

SR

DD

FF

FF

FR

SiE

$D D$, Desire for drinks; $E F$, Enjoyment of food; $E O E$, emotional overeating; $E U E$, emotional undereating; $F F$, food fussiness; $F R$, food responsiveness; $R$, item with reversed scoring; SiE, slowness in eating; $S R$, satiety responsiveness. 